\title{
Puzzles, Progress, Prospects: pre-Summary for the Quark Matter 2009 Conference
}

\author{
W.A. Zajc \\ Department of Physics, Columbia University, New York, NY, 10027, USA
}

\begin{abstract}
The new millennium's qualitative advances in relativistic heavy ion physics are in part due to, and are in part causing, a new appreciation for quantitative rigor in both experimental and theoretical work in the field. In these proceedings for the conference-opening "pre-Summary" talk I present an annotated guide to the figures and points made in my talk[1].
\end{abstract}

\section{Introduction}

The field of relativistic heavy ion physics was born in 1974, at a workshop hosted at the Bear Mountain conference center[2] in New York state. The discovery of QCD[3, 4] during the field's infancy 11 led to a precocious childhood ripe with the expectation of quark-gluon plasma formation[5] in high energy nuclear collisions. A not atypical adolescence followed, in which vaunting ambitions were confronted with life's realities (most notably the programmatic restrictions and/or prohibitions on obtaining quality reference and control data at LBL's Bevalac, BNL's AGS and CERN's SPS). With young adulthood has come maturity, a desire to "put away childish things", and a consolidation of results and discoveries that promises a long and fulfilling future. In what follows I will present a few of life's lessons that have been learned along the way.

\section{Know Your Reference}

In its most straightforward incarnation, experimental relativistic heavy ion physics is dedicated to the search for new phenomena not observed in the "baseline" measurements of $p+p$ nor in the "control" measurements of $\mathrm{p}+\mathrm{A}$ collisions. As alluded to above, this has been true for several fixed-target programs. As a case in point, consider the "DLS Puzzle", which refers to an excess seen in the invariant mass of $e^{+} e^{-}$pairs as measured by the Di-Lepton Spectrometer in low energy $(\sim 1 \mathrm{~A} \cdot \mathrm{GeV}) \mathrm{C}+\mathrm{C}$ and $\mathrm{Ca}+\mathrm{Ca}$ collisions 6 ] 2 . A significant excess of pairs in the invariant mass range $0.2<M\left(e^{+} e^{-}\right)<0.5 \mathrm{GeV}$ not accounted for by then extant transport models was observed, and later confirmed by the HADES collaboration[7]. Theoretical understanding of the "excess" followed from a corrected understanding of the critical role the $\Delta$ Dalitz decay and of bremsstrahlung production in $\mathrm{p}+\mathrm{n}$ collisions incorporated into a detailed transport code[8], and as verified by comparison to measured $\mathrm{p}+\mathrm{p}$ baseline and $\mathrm{p}+\mathrm{d}$ control measurements [9]. The

\footnotetext{
${ }^{1}$ Some liberty with strict temporal has been taken in the interest of poetic license.

${ }^{2} \mathrm{RHIC}$ and LHC students, please note that here energy is specified as the kinetic energy per nucleon of the beam. Preprint submitted to Nuclear Physics A 
clear lesson here is the importance of in situ measurements in the same channels in the same apparatus in simpler systems, and attention to the fine details (see also following section) when incorporating same into models: Know Your Reference.

\section{Details Matter}

The first two-pion HBT measurements at RHIC [14, 15] clearly demonstrated that $R_{\text {out }} / R_{\text {side }} \sim$ 1 , with the ratio showing only a weak dependence on the transverse momentum of the pair. This was in stark contrast to predictions [10, 11] that a first-order phase transition would result in $R_{\text {out }}$ being significantly larger than $R_{\text {side }}$, a result that was expected to persist even in the case of a relatively smooth cross-over transition[12]. Calculations using ideal hydrodynamics[13] produced excellent agreement with the single-particle transverse momentum spectra and of elliptic flow, but failed to describe the observed values of $R_{\text {out }}$ and $R_{\text {side }}$, leading to the "HBT Puzzle at RHIC".

A resolution to this issue is now at hand. Recent work by Pratt [16] has demonstrated that it is not one single factor, but rather a "conspiracy" of several factors that contributed to explaining the observed values of $R_{\text {out }}$ and $R_{\text {side }}$. These include viscous corrections, a more sophisticated treatment of final state interactions between the two pions, a stiffer equation of state and (perhaps most importantly, and with significant implications for other issues in the hydrodynamic description), pre-thermal acceleration of the matter[17]. The lesson here should be clear- while it is tempting to invoke schematic models involving exotic mechanisms to "resolve" the HBT puzzle, it is necessary first to analyze completely the (so-called) mundane effects neglected in the leading-order hydrodynamic description: Details Matter.

\section{Control Your Control Measurement}

No single theoretical prediction of a deconfinement signal has attracted more attention than the suggestion by Matsui and Satz[18] that the charmonium states would be suppressed in a quark-gluon plasma due to Debye screening 3 . Yet despite an extraordinary amount of theoretical and experimental activity, ambiguities remain[20]. Understanding the suppression pattern will required careful measurement of the feed-down from both $\chi_{C}$ 's and $B$-mesons; such efforts have begun at RHIC but have yet to reach quantitative precision. At energies above those of the SPS the effects of $c-\bar{c}$ coalescence [21, 22] must be considered, which in turn requires good knowledge of the $p_{T}$ and rapidity dependence of open charm production. Cold nuclear matter (CNM) have been carefully reconsidered at SPS energies and found larger than previously estimated, particularly when including the interplay with (anti)-shadowing[23]. At RHIC, CNM effects are essentially unconstrained by the statistical precision of the Run- $3 \mathrm{~d}+\mathrm{Au}$ data set, in that the errors on the break-up cross section are comparable to its magnitude, which in turn implies that one can not rule out the entire suppression pattern observed in $\mathrm{Au}+\mathrm{Au}$ collisions as being consistent with presence of CNM-effects only. While all of these issues are being or will be resolved by analysis of data sets with much greater integrated luminosity, two lessons seem inescapable: 1) The $\mathrm{J} / \Psi$ is a hadron with appreciable cross-section in nuclear matter and (even more importantly) 2) Control Your Control Measurement.

${ }^{3}$ This sentence is carefully worded to avoid a misstatement I made during my talk: The Matsui and Satz paper is not the most referenced paper in our field; Bjorken's paper on boost invariance [19] has significantly more references. I am grateful to Larry McLerran both for pointing this out to me and for doing so privately rather than publicly. 


\section{The Shape of Things to Come}

The title of this section is taken from H.G. Wells' evocative novel of the same name 24], but both in reading Wells' work and the remainder of this article, one should keep in mind Bohr's famous dictum "Prediction is very difficult, especially about the future."[25]. Therefore, in what follows work that postdates the presentation of my talk will be cited where useful.

\subsection{Event Morphology}

Five years ago Miklos Gyulassy's call for 10-dimensional 4 tomography of RHIC collisions [26] seemed at best fanciful. Recent work has brought us much nearer to that goal, although here I will focus more in morphology, that is, the study of event shapes, rather than tomography. The outstanding example of this is the "ridge", that is, the enhancement in particle production seen in nucleus+nucleus collisions on the near-side of a "trigger" particle. This feature extends at least for $\Delta \eta \sim \pm 4$ units of pseudo-rapidity about the "trigger" particle. Here the quotes on "trigger" are used to highlight the ambiguity (or ubiquity) of ridge phenomena[27]. Such enhancements have been observed in untriggered analyses[28], in events with moderately high triggers $\left(p_{T}^{T r i g}>2.5 \mathrm{GeV} / \mathrm{c}\right)[29]$, and in events with very high $p_{T}$ triggers[30]. Note that in the latter two cases, the ridge enhancement, while clearly present, is at most a few percent enhancement in yield over that the bulk soft particle production. Perhaps the most impressive morphological feature of the ridge is its extent over a huge range in relative pseudo-rapidity. Data on the relative extent in rapidity would be most welcome- it seems likely that the observed correlations are indeed in relative angle rather than in relative momentum. This, along with the fact that ridge enhancements are seen for all values of $p_{T}^{\text {trig }}$ has proven to be a very challenging to theoretical models; it is safe to say that no model is able to incorporate the ridge over the complete dynamic range[31].

\section{2. "Details Matter" Applied}

The realization that thermal radiation from a quark-gluon plasma is a key diagnostic actually predates the coinage of that name for deconfined quarks and gluons [32, 33]. But unambiguous detection of thermal photons, whether real or virtual, has proved maddeningly elusive. In the case of real photons, the difficulties are in large part due to the (nearly) overwhelming background of decay photons from $\pi^{0}$ 's and $\eta$ 's. Virtual pairs evade this background, but have subtle and demanding experimental challenges of their own, in which details truly do matter in determining and removing all sources of background, from both physics channels (internal conversions, correlated open charm production) and backgrounds (external conversions, decays). Both PHENIX [34] and NA60 [35, 36] have met those challenges, providing first data on thermal pairs from the medium in $\sqrt{s_{N N}}=200 \mathrm{GeV} \mathrm{Au}+\mathrm{Au}$ collisions and $\sqrt{s_{N N}}=17 \mathrm{GeV} \mathrm{In}+\mathrm{In}$ collisions, respectively. These results open a window on a critical new observable, which will need to be thoroughly explored in $\mathrm{p}+\mathrm{p}$ and $\mathrm{d}+\mathrm{A}$ collisions as well as in theoretical models.

\subsection{What's Past Is Prologue}

Just as the detection of relatively low transverse momentum thermal photons in relativistic heavy ion collisions has been a long-sought but recently realized goal, so has been the observation [37] of higher momentum, perturbatively produced direct photons. In principle, this

${ }^{4}$ Recent converts to the field please note that these dimensions are not those of Anti-de Sitter space. 
would seem to satisfy a desideratum famously expressed by Wilczek[38] in a Physics Today article published in 2000:

At the first level, one might hope to observe phenomena that are very difficult to interpret from a hadronic perspective but have a simple qualitative explanation based on quarks and gluons... But there is a second, more rigorous level that remains a challenge for the future. Using fundamental aspects of QCD theory ... one can make quantitative predictions for the emission of various kinds of "hard" radiation from a quark-gluon plasma. We will not have done justice to the concept of a weakly interacting plasma of quarks and gluons until some of these predictions are confirmed by experiment.

It is striking to consider the evolution of our thinking from that time. It is indeed true that the production rate of direct photons is consistent with $\mathrm{PQCD}$ calculations, providing both a verification of the "binary collision counting" paradigm and an important baseline establishing the validity of the jet quenching results. But at the same time these very phenomena are not interpreted as establishing the presence of a "weakly interacting plasma", but rather are important elements in establishing the strongly-coupled quark-gluon plasma (sQGP).

There is an important third, potentially even more rigorous level in Wilczek's hierarchy of observables: differential measurements that extract quantitative results for transport coefficients or medium modifications. An excellent example- "tagged" photon-hadron correlations to determine medium modifications to the fragmentation functions[39]- was proposed several years before Wilczek's Physics Today article. The purity of the method, which relies on the transparency of the medium to the photon to establish the partonic $Q^{2}$, is offset by the rate penalty 5 exacted by the additional power of $\alpha_{E M}$. Only now are first results becoming available from measurements using this technique [40], but it promises to be an important quantitative tool for future RHIC measurements and an essential element of the LHC program.

\subsection{Towards True Jet Quenching}

It has become common practice to refer to the discoveries of strong suppression of high $p_{T}$ particles at RHIC energies [41] and the disappearance of the away-side "jet"[42] as "jet quenching", a term coined by Gyulassy and Plumer[44] with due reference to Bjorken's numerically incorrect but nonetheless seminal initial work on the topic[43]. Of course the leading particles used to date to study suppression are not jets; they are used as a stand-in for same due to the difficulties in detecting jets (especially with traditional jet algorithms) in an environment with a high multiplicity of soft particles. These difficulties arise because even statistically unlikely Poisson fluctuations in the emission pattern of soft and moderate $p_{T}$ hadrons that can mimic a jet's localized energy production are more probable than the probability of a high- $p_{T}$ scatter. This issue has been understood for some time [45]; for example the fine segmentation of the PHENIX calorimeter $\Delta \eta \times \Delta \phi \sim 0.01 \times 0.01$ was driven by the requirement of isolating high- $p_{T}$ leading particles from the soft background.

Recently, progress has been towards true jet reconstruction at RHIC. This has been made possible in part simply by higher integrated luminosities providing access to higher energy jets- the

\footnotetext{
${ }^{5}$ As an example, RHIC running at 5 times design luminosity produces about one $15 \mathrm{GeV}$ photon per hour in the PHENIX central arms, which must be combined with a penalty factor of $10^{-(2-3)}$ for measuring the complete range of the fragmentation function.
} 
soft background fluctuations have a limited reach in fake jet energy (making this effect almost irrelevant at LHC energies). But there have also been new developments in algorithms specifically designed for jet reconstruction in the heavy ion environment[46]. Comparing results presented at this conference from two rather different approaches to the problem[47, 48] should be very useful for understanding any remaining systematic uncertainties in extracting the jet signal from the background, and for then determining jet modifications in deconfined nuclear matter.

\subsection{Quantifying Jet Quenching}

The difficult task of measuring the modification of jets and/or high- $p_{T}$ hadron production in nuclear collisions is motivated by the prospect of extracting quantitative information on the transport properties of the medium. This possibility in turn follows from the ability to calculate the primary production rate using perturbative $\mathrm{QCD}(\mathrm{pQCD})$, and from $\mathrm{pQCD}$ calculations of energy loss in the medium, typically assumed to be homogenous or following a simply parameterized 1-d expansion. Comparison with actual heavy ion data requires a much more sophisticated treatment of the time development. Recently, a systematic study of three models of jet quenching has been performed by embedding their microscopic prescriptions in a realistic 3-d hydrodynamic model of the expanding medium[49]. A gratifying level of agreement is achieved by all of the models to the single-particle suppression measure $R_{A A}\left(p_{T}\right)$, at the expense of a frustratingly large range 6 in the associated values of the quenching parameter $\hat{q}$. To some extent this is an illustration of the "fragility" of single-observables [50], borne out by the predicted differences of the same three models in computing more differential observables such as the dependence of the suppression with respect to the reaction plane. However, equally important is a consistent treatment of effects such as collisional as well as radiative terms, assumed value of the coupling 'constant', kinematic cut-offs, assumed scale hierarchies, etc. [51, 52]. Both the work noted above and the efforts of the TECHQM[53] collaboration have been instrumental in highlighting these issues and in enabling their resolution.

\subsection{Hydrodynamics for the New Millennium}

The seemingly staid topic of relativistic fluid dynamics has been revolutionized in the past decade, driven in large part by the need to describe the near-perfect fluidity observed at RHIC. In particular, the desire to compare realistic hydrodynamic calculations with non-zero viscosity to the experimental data necessitated a complete re-examination of the formalism. While it has been known for some time that limiting the expansion to first-order in the gradients led to severe instabilities [54], the various second-order expansions were known to be stable and thought to be complete. This has turned out not be the case; previous workers had made assumptions appropriate for astrophysical applications that led to the dropping of terms that are important in a completely relativistic system such as the RHIC fluid. Valuable guidance in this exercise was obtained from the Ads/CFT correspondence, at least for the case of conformal fluids[55].

The end result of this effort, combined with extensive work on numerical implementation of the resulting equations, has been a "concordance" in modeling RHIC collisions with secondorder dissipative hydrodynamics [56]. Impressive agreement with a wide variety of bulk observables has been obtained[57, 58, 59], leading to the exciting prospect of extracting numerical

\footnotetext{
${ }^{6} \mathrm{~A}$ factor of $\sim 4$ across these three models; inclusion of other models would extend this range to essentially a factor of 10 .
} 
values of transport coefficients. A more difficult task will be determining the systematic uncertainties in such extractions due to ill-determined initial conditions, eccentricity fluctuations and hadronic rescattering, but the rate of recent progress supports cautious optimism that these challenges will be met in the near future.

\subsection{Knowing Knudsen}

While each transport coefficient provides insight into the medium, the ratio of shear viscosity $\eta$ to entropy density $s$ has attracted far and away the most attention, in very large part due to the bold conjecture [60] that this ratio satisfies a "quantum bound" $\eta / s \geq 1 / 4 \pi$ (in natural units). Both the detailed hydrodynamics simulations mentioned above and a variety of heuristic estimates $61,62,63,64$ suggest that $\eta / s \sim(1-4) / 4 \pi$ for the RHIC fluid, making it the most perfect of all imperfect fluids studied in the laboratory. This provocative statement, while supported by arguments by Danielewicz and Gyulassy[65] which anticipated the bound on $\eta / s$, clearly demands strong scrutiny in order to establish its validity.

An intriguing recent development has been the parametrization of deviations in the elliptic flow pattern from that of ideal hydrodynamics in terms of the Knudsen number $K \equiv \lambda / R$, where $\lambda$ is the mean free path and $R$ is a characteristic system size[64]. Specifically, the centrality dependence of the elliptic flow parameter $v_{2}$ scaled by the (estimated) initial state eccentricity $\epsilon$ is assumed to have the simplest linear form consistent with ideal hydrodynamics in the limit $K \rightarrow 0$. A fit to the experimental data produces a value for $\lambda$, which is then used to determine the viscosity under the assumption of Boltzmann transport. The resulting values for $\eta / s$ span a range of 0-10 times the quantum bound[66], a range driven in large part from extreme sensitivity to the assumptions of the ill-determined initial state eccentricity.

There is an interesting dialectic at play here. While it is tempting to identify the striking pattern of constituent-quark scaling [67,68] as evidence for underlying quasi-particles, the existence of long-lived quasi-particles is inconsistent with a strongly coupled medium near the quantum bound [69], this result being well-known in condensed matter physics [70]. Conversely, hydrodynamics calculations, which correctly forgo particle transport during the (nearly perfect) fluid phase, often suffer from failing to treat the true particles present in the hadronic phase. Somewhat surprisingly, the determinations of $\eta / s$ based on Knudsen number parameterizations, with their implicit assumption of ballistic transport of quasi-particles, are not vastly different from those as determined from second-order relativistic hydrodynamic calculations incorporating viscous effects[72]. This may be viewed as a first step towards quantifying the many systematic uncertainties [71] in the Knudsen number approach, but there remains more work to be done. As but one example, it will be important to know how realistic it is to identify a single value for $\eta / s$ to represent the time evolution of this key quantity in the course of a heavy ion collision.

\subsection{Critical Developments}

There is general agreement that the predicted first-order phase transition at high net baryon densities between hadronic and deconfined matter should end at a critical point [73]. This conclusion is dependent on a proper treatment using the correct $u, d$ and $s$ physical quark masses, and there is still very considerable uncertainty in the location of the critical point in the $\left(T, \mu_{B}\right)$ plane [74]. Similarly, while it is expected that there will be "strong" fluctuations in the "vicinity" of the critical point, much work remains to sharpen these statements to the point where hypotheses can be rejected by observation. The situation is not dissimilar to previous stages in our field's history, which teaches us that qualitative advances in experimental data are necessary 
for progress. Ongoing analyses of SPS data, recent low-energy results from RHIC[75] and ambitious future plans [76, 77] will provide that impetus towards extending our understanding of the QCD phase diagram.

\section{Conclusions}

Our field is suffering, in the most pleasant sense of the word, from an embarrassment of riches. The enormous progress made in the past decade will be sustained into the future by new low-energy facilities; by upgraded experiments, luminosity and capabilities at RHIC; and by the unprecedented increase in center-of-mass of energy and sophisticated experiments of the LHC, making this truly is a golden era in relativistic heavy ion physics.

\section{Acknowledgments}

This work was supported by U.S. Department of Energy grant DE-FG02-86ER40281.

\section{References}

[1] http://qm09.phys.utk.edu/indico/getFile.py/access?contribId=491\&amp; sessionId=25\&amp; res Id=0\&amp; materialId=sli

[2] Report of the workshop on BeV/nucleon collisions of heavy ions how and why, Bear Mountain, New York, Nov. 29 Dec. 1, 1974 (BNL-AUI, 1975).

[3] D. J. Gross and F. Wilczek, Phys. Rev. D 8, 3633 (1973).

[4] H. D. Politzer, Phys. Rev. Lett. 30, 1346 (1973).

[5] E. V. Shuryak, Phys. Rept. 61, 71 (1980).

[6] R. J. Porter et al. [DLS Collaboration], Phys. Rev. Lett. 79, 1229 (1997) [arXiv:nucl-ex/9703001].

[7] I. Frohlich et al. [HADES collaboration], Int. J. Mod. Phys. A 24, 317 (2009) [arXiv:0809.2764 [nucl-ex]].

[8] E. L. Bratkovskaya and W. Cassing, Nucl. Phys. A 807, 214 (2008) [arXiv:0712.0635 [nucl-th]].

[9] W. K. Wilson et al. [DLS Collaboration], Phys. Rev. C 57, 1865 (1998) [arXiv:nucl-ex/9708002].

[10] S. Pratt, Phys. Rev. D 33, 1314 (1986).

[11] G. Bertsch, M. Gong and M. Tohyama, Phys. Rev. C 37, 1896 (1988).

[12] D. H. Rischke and M. Gyulassy, Nucl. Phys. A 608, 479 (1996) [arXiv:nucl-th/9606039].

[13] U. W. Heinz and P. F. Kolb, Nucl. Phys. A 702, 269 (2002) [arXiv:hep-ph/0111075].

[14] C. Adler et al. [STAR Collaboration], Phys. Rev. Lett. 87, 082301 (2001) [arXiv:nucl-ex/0107008].

[15] K. Adcox et al. [PHENIX Collaboration], Phys. Rev. Lett. 88, 192302 (2002) [arXiv:nucl-ex/0201008].

[16] S. Pratt, Phys. Rev. Lett. 102, 232301 (2009) [arXiv:0811.3363 [nucl-th]].

[17] J. Vredevoogd and S. Pratt, arXiv:0810.4325 [nucl-th].

[18] T. Matsui and H. Satz, Phys. Lett. B 178, 416 (1986).

[19] J. D. Bjorken, Phys. Rev. D 27, 140 (1983).

[20] See the contribution of L. A. L. Levy to these proceedings for a detailed review: arXiv:0908.2361 [nucl-ex].

[21] P. Braun-Munzinger and J. Stachel, Phys. Lett. B 490, 196 (2000) [arXiv:nucl-th/0007059].

[22] R. L. Thews, M. Schroedter and J. Rafelski, Phys. Rev. C 63, 054905 (2001) [arXiv:hep-ph/0007323].

[23] E. Scomparin and f. t. N. collaboration, arXiv:0907.3682 [nucl-ex].

[24] http://en.wikipedia.org/wiki/The_Shape_of_Things_to_Come

[25] http://www.quotationspage.com/quote/26159.html

[26] M. Gyulassy, arXiv:nucl-th/0403032.

[27] See http://qgp.phy.duke.edu/2008/05/20/a-new-angle-on-the-ridge/ for an informal discussion on the ridge.

[28] J. Adams et al. [STAR Collaboration], Phys. Rev. C 73, 064907 (2006) [arXiv:nucl-ex/0411003].

[29] B. Alver et al. [PHOBOS Collaboration], arXiv:0903.2811 [nucl-ex].

[30] :. B. I. Abelev [The STAR Collaboration], arXiv:0909.0191 [nucl-ex].

[31] See the contribution of J.L. Nagle to these proceedings for a detailed review of ridge phenomena and theoretical models of same: J. L. Nagle, arXiv:0907.2707 [nucl-ex].

[32] E. L. Feinberg, Nuovo Cim. A 34, 391 (1976). 
[33] E. V. Shuryak, Phys. Lett. B 78, 150 (1978) [Sov. J. Nucl. Phys. 28, 408.1978 YAFIA,28,796 (1978 YAFIA,28,796808.1978)].

[34] A. Adare et al. [PHENIX Collaboration], arXiv:0804.4168 [nucl-ex].

[35] R. Arnaldi et al. [NA60 Collaboration], Eur. Phys. J. C 59, 607 (2009) [arXiv:0810.3204 [nucl-ex]].

[36] R. Arnaldi et al. [NA60 Collaboration], Phys. Rev. Lett. 102, 222301 (2009) [arXiv:0812.3100 [nucl-ex]]

[37] S. S. Adler et al. [PHENIX Collaboration], Phys. Rev. Lett. 94, 232301 (2005) [arXiv:nucl-ex/0503003].

[38] F. Wilczek, QCD made simple, Phys. Today 53N8, 22 (2000).

[39] X. N. Wang, Z. Huang and I. Sarcevic, Phys. Rev. Lett. 77, 231 (1996) [arXiv:hep-ph/9605213].

[40] A. Adare et al. [PHENIX Collaboration], Phys. Rev. C 80, 024908 (2009) [arXiv:0903.3399 [nucl-ex]].

[41] K. Adcox et al. [PHENIX Collaboration], Phys. Rev. Lett. 88, 022301 (2002) [arXiv:nucl-ex/0109003].

[42] C. Adler et al. [STAR Collaboration], Phys. Rev. Lett. 90, 082302 (2003) [arXiv:nucl-ex/0210033].

[43] J. D. Bjorken, "Energy Loss Of Energetic Partons In Quark - Gluon Plasma: Possible Extinction Of High $p_{T}$ Jets In Hadron-Hadron Collisions," FERMILAB-PUB-82-059-THY, (1982). Note that while Bjorken used the term "jet extinction", he also states "This is quite sufficient to quench low- $p_{T}$ jets!!".

[44] M. Gyulassy and M. Plümer, "Jet Quenching In Dense Matter," Phys. Lett. B 243, 432 (1990).

[45] See for instance the "Jet Quenching" slide available from http://wWw.phenix.bnl.gov/phenix/WWW/publish/zajc/sp/presentations/BL available separately as http://wWw.phenix.bnl.gov/phenix/WWW/publish/zajc/sp/presentations/BUColloquium/Html/img033.jp

[46] Y. S. Lai and B. A. Cole, arXiv:0806.1499 [nucl-ex]

[47] S. Salur, arXiv:0907.4536 [nucl-ex].

[48] Y. S. Lai for the PHENIX Collaboration, arXiv:0907.4725 [nucl-ex].

[49] S. A. Bass, C. Gale, A. Majumder, C. Nonaka, G. Y. Qin, T. Renk and J. Ruppert, Phys. Rev. C 79, 024901 (2009) [arXiv:0808.0908 [nucl-th]].

[50] K. J. Eskola, H. Honkanen, C. A. Salgado and U. A. Wiedemann, Nucl. Phys. A 747, 511 (2005) [arXiv:hep$\mathrm{ph} / 0406319]$

[51] "Hard Probes: Past, Present, and Future", B.A. Cole, talk presented at Quark Matter 2008, http://www.veccal.ernet.in/ pmd/qm2008/webpage/Program/10Feb/bcole.pdf

[52] S. Jeon, arXiv:0907.4691 [nucl-th].

[53] https://wiki.bnl.gov/TECHQM/index.php/Partonic_Energy_Loss.

[54] W. A. Hiscock and L. Lindblom, Phys. Rev. D 31, 725 (1985).

[55] R. Baier, P. Romatschke, D. T. Son, A. O. Starinets and M. A. Stephanov, JHEP 0804, 100 (2008) [arXiv:0712.2451 [hep-th]].

[56] See TECHQM "Bulk Evolution" pages https://wiki.bnl.gov/TECHQM/index.php/Bulk_Evolution in particular Summary of Workshops on Hydrodynamics at BNL, F. Karsch et al., May 14, 2008, available as https://wiki.bnl.gov/TECHQM/images/c/c1/Summary.pdf.

[57] P. Romatschke and U. Romatschke, Phys. Rev. Lett. 99, 172301 (2007) [arXiv:0706.1522 [nucl-th]].

[58] H. Song and U. W. Heinz, Phys. Rev. C 77, 064901 (2008) [arXiv:0712.3715 [nucl-th]].

[59] M. Luzum and P. Romatschke, arXiv:0901.4588 [nucl-th].

[60] P. Kovtun, D. T. Son and A. O. Starinets, Phys. Rev. Lett. 94, 111601 (2005) [arXiv:hep-th/0405231].

[61] S. Gavin and M. Abdel-Aziz, Phys. Rev. Lett. 97, 162302 (2006) [arXiv:nucl-th/0606061].

[62] R. A. Lacey et al., Phys. Rev. Lett. 98, 092301 (2007) [arXiv:nucl-ex/0609025].

[63] A. Adare et al. [PHENIX Collaboration], Phys. Rev. Lett. 98, 172301 (2007) [arXiv:nucl-ex/0611018].

[64] H. J. Drescher, A. Dumitru, C. Gombeaud and J. Y. Ollitrault, Phys. Rev. C 76, 024905 (2007) [arXiv:0704.3553 [nucl-th]].

[65] P. Danielewicz and M. Gyulassy, Phys. Rev. D 31, 53 (1985).

[66] A. Tang, arXiv:0907.4513 [nucl-ex].

[67] S. S. Adler et al. [PHENIX Collaboration], Phys. Rev. Lett. 91, 182301 (2003) [arXiv:nucl-ex/0305013].

[68] J. Adams et al. [STAR Collaboration], Phys. Rev. Lett. 92, 052302 (2004) [arXiv:nucl-ex/0306007].

[69] L. A. Linden Levy, J. L. Nagle, C. Rosen and P. Steinberg, Phys. Rev. C 78, 044905 (2008) [arXiv:0709.3105 [nucl-th]].

[70] S. Sachdev and M. Mueller, arXiv:0810.3005 [cond-mat.str-el].

[71] J.L. Nagle, P. Steinberg, and W.A. Zajc, arXiv:0908.3684 [nucl-th].

[72] H. Masui, J. Y. Ollitrault, R. Snellings and A. Tang, arXiv:0908.0403 [nucl-ex].

[73] M. A. Stephanov, K. Rajagopal and E. V. Shuryak, Phys. Rev. Lett. 81, 4816 (1998) [arXiv:hep-ph/9806219].

[74] For a recent review see Z. Fodor and S. D. Katz, arXiv:0908.3341 [hep-ph].

[75] J. Adams et al. [STAR Collaboration], "Identified particle production, azimuthal anisotropy, and interferometry measurements in $\mathrm{Au}+\mathrm{Au}$ collisions at $\sqrt{s_{N N}}=9.2 \mathrm{GeV}$," to be submitted to Physical Review $\mathbf{C}$.

[76] J. M. Heuser [CBM Collaboration], arXiv:0907.2136 [nucl-ex].

[77] V. Toneev, PoS C POD07, 057 (2007) [arXiv:0709.1459 [nucl-ex]]. 